\title{
Urgences
}

\section{Les grands départs ou les retours en écho}

\section{Renald Bérubé}

Numéro 23, avril 1989

Lisière du livre

URI : https://id.erudit.org/iderudit/025522ar

DOI : https://doi.org/10.7202/025522ar

Aller au sommaire du numéro

Éditeur(s)

Urgences

ISSN

0226-9554 (imprimé)

1927-3924 (numérique)

Découvrir la revue

Citer cet article

Bérubé, R. (1989). Les grands départs ou les retours en écho. Urgences, (23),

121-132. https://doi.org/10.7202/025522ar d'utilisation que vous pouvez consulter en ligne.

https://apropos.erudit.org/fr/usagers/politique-dutilisation/ 


\title{
RENALD BÉRUBÉ \\ Les grands départs ou les retours en écho
}

\author{
À Yolande et Jacques Languirand, \\ pour les si nombreuses rencontres, \\ discussions, etc. avec Thérèse et moi.
}

HECTOR - Au milieu de la pièce. Comme s'il faisait des essais de voix.
Hector! Hector! Puis, de l'autre côté. Hector! Hector! [...] Écoute... (...)
L'écho... Idem. Hector! Hector! A Margot (sa femme). Ma voix résonne
comme dans une gare! La maison est pleine de trous, de poches d'air... Ça
donne le vertige! Les grands départs, p. 15-16.

MARGOT - Tu t'écoutes parler.

HECTOR - Je suis mon plus fidèle auditeur. Mais j'écoute aussi le monde. lbid., p. 22.

MARGOT - Je t'en prie! Sophie! Où es-tu?

HECTOR - Imitant l'écho. Tu... tu...

MARGOT - Tu as bientôt fini de faire le perroquet?

Ibid., p. 25.

On le sait assez bien, merci, depuis Freud et Marthe Robert en particulier, le roman des origines, qui est aussi l'origine du roman', maintient toujours ses droits. L'importance du commencement, romanesque ou autre, étant ainsi incontournable, aussi bien commencer par icelui. Même (surtout?) s'il s'agit d'une pièce de théâtre - a play, en anglophonie - dans laquelle par ailleurs le familial, c'est-à-dire l'originel, joue un rôle déterminant, même en l'absence de tout nom de famille.

Première pièce publiée de Jacques Languirand, Les grands départs $^{2}$, créée à la télévision de Radio-Canada dans une réalisation de Louis-Georges Carrier le 1er octobre 1957, ne fut pas la première pièce jouée de l'auteur, mais bien plutôt la quatrième. Au commencement, le 9 mars 1956, il y eut Les insolites, créée par La Compagnie de Montréal «à l'occasion du Festival d'Art dramatique de l'Ouest du Québec dans une mise en scène de Guy L'Écuyer, un décor de Jean-Claude Rinfret, des costumes de Yolande Delacroix-Pelletier et avec une musique originale d'André Mathieu " ${ }^{3}$. À l'occasion des étapes régionale puis nationale du Festival, la pièce de Languirand s'adjugea moult prix et trophées, dont celui de «la meilleure pièce canadienne " ${ }^{4}$, si bien que, le Festival terminé, Languirand et La Compagnie de Montréal s'installent au théâtre Anjou où seront données, pendant plus d'un mois, des représentations de la pièce. 
À l'automne de 1956, Languirand fonde Le Théâtre de Dix Heures (1300, rue Saint-Urbain); c'est là que, le 1er novembre 1956, sera créée sa deuxième pièce, Le roi ivre, farce en deux tableaux jouée par la troupe «Les Insolites» dans une mise en scène de Louis-Georges Carrier. Le roi ivre étant une pièce brève, le programme mettait aussi une autre pièce à l'affiche: On demande un souvenir, de Louis-Georges Carrier.

Une vingtaine de jours après la création du Roi ivre, soit le 22 novembre 1956, à 22 heures, première création d'une pièce de Languirand à la (jeune) télévision de Radio-Canada: Hamlet, adaptation d'après Thomas Kyd, Louis-Georges Carrier étant le réalisateur. Hamlet d'après Thomas Kyd et non d'après Shakespeare - il y a là, faut-il le dire, quelque chose d'étrange, d'insolite. Thomas Kyd (1558-1594) a (donc!) pourtant bel et bien existé; il est l'auteur de La tragédie espagnole (vers 1586-1587), pièce d'une importance et d'un succès considérables dans l'histoire du théâtre élisabéthain, et on lui attribue généralement un Hamlet qui fut représenté à Londres dès 1589 ou même avant, soit plus d'une dizaine d'années avant celui de Shakespeare. Il y a pourtant un hic: le Hamlet attribué à Kyd est une pièce depuis fort longtemps perdue ${ }^{5}$. Alors? Languirand n'allait pas de sitôt quitter l'insolite ou vice versa: il anime, à la radio de Radio-Canada, une émission intitulée Le dictionnaire insolite (réalisateur: Lorenzo Godin) qui, pour la saison 1956-1957, se méritera le trophée Laflèche pour la plus importante contribution à la radio canadienne d'expression française. Et l'émission sera à l'origine du livre du même titre que publiera plus tard Languirand ${ }^{6}$. A création insolite, auditoire perplexe pourrait-on dire: Languirand animera aussi en 1957, sorte d'auditeur ou de lecteur de lui-même, Les carnets de Monsieur Perplex à la radio de Radio-Canada - et Perplex sera le héros, interprété par Languirand lui-même, de sa pièce Le gibet créée le 10 novembre $1958^{7}$. Mais voilà que tel un Hamlet perplexe nous nous devançons - il s'agissait de l'avant Les grands départs, non de l'après.

Les grands départs, donc: première pièce publiée de Languirand, elle fut la quatrième à être créée, la deuxième à la télévision radio-canadienne. Evénement qui eut lieu le 1er octobre 1957, moins d'un an après la création du Hamlet, et dans une réalisation de Louis-Georges Carrier, bien sûr. Pourtant cette pièce qui, à notre connaissance, n'a jamais été jouée sur une scène montréalaise par une troupe professionnelle mais doit finalement l'être par le Théâtre d'Aujourd'hui à l'automne 1989, fut «d'abord conçue pour le théâtre», nous dit-on à la page 6 de sa première édition. 
Mais voilà, nous nous devançons encore; lecture paratextuelle obligeant, il convient de commencer ladite lecture des Grands départs par le commencement, c'est-à-dire par la lecture de sa couverture, occasion du premier contact du lecteur ou de la lectrice avec l'objet à lire. Et si on avait entendu parler de l'objet avant de le voir ou de le lire? Les romans des origines ne sont jamais aussi simples qu'on pourrait d'abord le croire; d'autant plus que Les grands départs, à proprement parler, n'a pas de couverture, pas vraiment.

Donc, vous n'avez pas vu Les grands départs de Languirand lors de la création de la pièce à la télévision; vous en avez bien entendu parler, mais vous ne l'avez pas encore lue. Ce que vous allez faire, dans l'édition originale empruntée d'une bibliothèque puisqu'elle n'est plus disponible en librairie. Lecture faite, vous refermez le livre, laissez mijoter votre lecture tout en tenant le livre entre vos mains ou en le regardant, déposé là sur votre table de travail. Et puis, vous commencez à le refeuilleter avant de recommencer à le feuilleter très sérieusement.

Les grands départs, si vous devez vous résumer le texte que vous venez de lire - et le résumé est bien lui-même une pratique transtextuelle, hypertextuelle plus précisément ${ }^{8}$-, est doric une pièce en trois actes qui se déroulent en un seul lieu et en une seule nuit. Prétexte de l'action (ou de l'inaction): on attend les déménageurs qui doivent assurer le transport du mobilier et autres effets de l'appartement «modeste» (p. 14) que l'on quitte pour aller habiter un «appartement plus petit» (p. 20). On, c'est-à-dire cinq personnes: Hector, son épouse Margot et leur fille Sophie, Eulalie, soeur de Margot, et le Grand-Père paralytique, père de Margot et d'Eulalie. L'acte I (p. 15-50), de manière classique, nous a mis au courant de la situation: la famille doit déménager dans un appartement plus petit parce qu'elle mangue d'argent (p. 20). Écrivain qui a «commencé trois ou quatre romans dans lesquels le personnage principal est un paralytique" et qui s'est toujours «arrêté un peu avant la fin du premier chapitre" (p. 19), Hector aura épuisé les économies de sa belle-famille (p. 19): celles de sa femme, celles de son beau-père, d'ailleurs "devenu paralytique à la suite d'une querelle avec (lui]» (p. 17), celles d'Eulalie sa belle-soeur à qui il a vendu l'idée de consacrer sa vie à son père (p. 46). Puisqu'il «faut avoir le courage de ne pas s'avorter" et "prendre la situation en main» (p. 21), les discussions seront féroces, désespérées et grotesques, entre les membres de la famille - y compris le Grand- 
Père qui, depuis quelque part «parmi les bagages» (p. 17), émet à diverses reprises une «plainte déchirante» (p. 23, 29, etc.). Quand, à la fin de l'acte, la sonnerie de l'entrée se fait entendre, on croit bien qu'il s'agit des déménageurs; mais à la vue de l'homme qui entre dans l'appartement, Eulalie s'évanouit (p. 50).

Le début de l'acte II (p. 53-79) nous a bientôt appris qu'il s'agit d'Albert, ancien ami d'Hector (p. 60), jadis prétendant d'Eulalie (p. 58 ), et que Margot même a pu regarder d'un oeil intéressé (p. 59). II a "fait le tour du monde" (p. 60) et revient vers Eulalie pour l'arracher à sa famille, puisque «la vie commence à cinquante ans" (p. 56). Albert aura été absent pendant «vingt ans» (p. 73), mais Eulalie partira tout de même avec lui (p. 72), Sophie, quant à elle, ira au "cinéma» (p. 77). Décisions prises, toutes deux, dans un climat d'affrontement et de règlements de comptes familiaux. «Si je comprends bien", dit Hector vers la fin de l'acte, «l'idée des grands départs fait son chemin» (p. 79).

L'acte III (p. 83-119) ou celui des petits retours déçus. Retour d'Eulalie, d'abord, qui a compris qu'elle entrait dans le monde et qui a eu peur (p.94) parce qu'elle n'avait «pas du tout imaginé que ça se passerait de cette façon» (p. 95): Albert, fatigué, a trouvé rapidement une chambre d'hôtel, «il portait de grosses bretelles» (p. 97), et lui a avoué qu'il n'avait «jamais pu faire le tour du monde" - "C'est tout. Il a détruit mon beau rêve» (p. 98). Elle aura "vécu à l'extérieur un peu moins d'une heure» (p. 99). Retour de Sophie ensuite: alors qu'elle était «prête à partir avec lui» (p. 110), son ami ne l'aura finalement amenée qu'au cinéma, qu'elle aura quitté sans attendre la fin du film (p. 108). Maintenant que «(t)oute la petite famille est là» (p. 107) de nouveau, comme au début, que «[I]'épreuve de la purgation" (p. 112) - épreuve aristotélicienne, cela va de soi: nous sommes au théâtre! - a eu lieu, le temps quotidien peut reprendre ses droits: «J'ai demandé l'heure parce qu'il est réconfortant de savoir, de temps à autre, l'heure qu'il est » (p. 111), chacun peut retrouver sa petite boule dans la gorge ( $p$. 116 ) et ses vieux vêtements (p. 117). Sauf Grand-Père qui, après avoir émis une plainte, fait tomber valise et boîte au milieu desquelles il se trouve, se lève, les regarde tous longuement, «repousse violemment Margot et Hector», et sort avec «une petite valise [...] en refermant violemment la porte». "ll nous méprise», dit Hector (p. 118-119).

Voilà le scénario qui se formule dans votre tête alors que vous refeuilletez la pièce, vous attardant sur les soulignés de votre première lecture. L'un des scénarios. Car vous avez déjà noté 
aussi des éléments d'ordre plus analytique: par exemple, que Hector est une sorte de grand maître de la parole provocatrice et insolite, paradoxale, alors que ses personnages romanesques préférés sont des paralytiques et que ses romans n'aboutissent pas, avortent; que le Grand-Père est devenu paralytique à la suite d'une querelle avec ledit grand maître et qu'il est le seul personnage à quitter pour de bon l'appartement en désordre, la gare résonnante pleine de poches d'air, à donner le vertige. Le seul en tout cas qui ne revienne pas tel l'écho, la pièce se terminant sur son départ. Et vous vous dites tout cela en pensant aussi aux avantpropos et aux photos qui accompagnent l'édition de la pièce, en jetant des coups d'oeil sur la maquette (de couverture?) qui s'offre au regard, le livre étant refermé.

La maquette, en dépit de la formule consacrée, n'est pas celle de la couverture. La couverture des Grands départs consiste en un mince carton uniformément blanc, découpé et replié selon le format et l'épaisseur du livre, et collé aux feuillets cousus de l'édition. La maquette est celle de la jaquette ${ }^{9}$, formule moins consacrée (plus insolite?) — «... la jaquette (ou liseuse)», écrit Gérard Genette ${ }^{10}$. Alors, flairant peut-être une piste intéressante, minutie et précision obligeant, vous avez recours au Grand Robert (tome IV, 1963):

LISEUR, EUSE, n. (Xlle s.; de lire)

I. 1. Celui, celle qui a l'habitude de lire beaucoup. V. Lecteur.

2. Technol. Ouvrier, ouvrière qui effectue le lisage (V. Lire 1, dér.).

II. LISEUSE, n.f. (XIXe s). Couteau à papier servant aussi de signet.

- Petite table à plusieurs tablettes, pour poser livres et revues (Cf.

fourre-tout, cit. 1). - Couvre-livre interchangeable. Liseuse en cuir, en tissu. - Vêtement de femme, sorte de petite veste en laine tricotée, pour lire au lit.

Suivant le guide, vous allez donc à Lire 1, dér.:

Lisage. n.m. (1785). Technol. Opération qui consiste à lire, à analyser un dessin pour tissu et à percer de trous disposés correctement les cartons qui sont montés dans le métier. Machine effectuant cette opération. -- Liseur, liseuse, lisible.

Voilà: lecteur ou lectrice assidu-e, vous savez désormais (par expérience, par définition) que le travail de lecture est aussi travail de lisage, c'est-à-dire d'analyse du dessin (et du dessein). Que la jaquette/liseuse est un couvre-livre interchangeable - Genette parle de son caractère "amovible», "comme constitutivement éphémère, qui invite presque le lecteur à s'en débarrasser une fois remplie (sa) mission d'affiche, et éventuellement de protection" "11 
- comme ce papier brun dont jadis vous (votre mère) recouvriez vos livres de petite école afin de les protéger, que les rabats de ladite jaquette peuvent bien, nouveaux couteaux à couper les pages, faire office de signets; qu'enfin, la liseuse/vêtement peut aussi, au lit, habiller les hommes, l'unisexe vestimentaire pouvant trouver là une application douillette pour mâles lecteurs, pourquoi pas.

Mais lisant toujours et le mieux possible, vous vous dites qu'il y a là davantage: et si la jaquette/liseuse effectuait elle-même, technol., un travail de lisage? D'autant plus que la jaquette des Grands départs «a été conçue par Jean-Claude Rinfret» nous dit le deuxième rabat de celle-ci, le même qui avait signé, nous l'avons vu, le décor des Insolites. Le "métier», pour employer un mot utilisé dans la définition de «lisage», a bel et bien ici ses droits: la jaquette comme maquette d'un décor possible de la représentation de la pièce; la jaquette, donc, comme lecture de cette pièce. Liseur ou liseuse en paratexte, il s'agit alors de lire, d'analyser ce paratexte déjà lui-même métatextuel, c'est-à-dire analytique et critique $^{12}$.

La jaquette des Grands départs n'utilise que le noir et blanc ainsi que diverses teintes de dégradés menant l'un à l'autre. Le recto et le verso (la première et la quatrième de... couverture?) se présentent à la fois comme le positif et le négatif et comme la reproduction en miroir d'une même photo: là où il y a noir et blanc au recto, il y a blanc et noir au verso, les divers dégradés suivant le même procédé; et «LES GRANDS DÉPARTS» imprimés en majuscules blanches et sur deux lignes allant, cela va de soi, de gauche à droite au recto, se liront symétriquement en noir et de droite à gauche, à l'envers, au verso. Du recto au verso, deux éléments sont disparus: le nom de l'auteur, écrit au recto en petites capitales blanches au-dessus du titre de la pièce, et le nom de l'éditeur écrit de bas en haut en plus petites capitales blanches dans le coin inférieur droit du recto de la jaquette. Le nom de l'auteur, en capitales noires identiques (sur fond blanc) aux blanches du recto et se lisant du bas vers le haut, est par ailleurs le seul élément à apparaître sur l'épine (de la jaquette): comme si cet élément était celui à afficher lorsque le livre se trouve debout sur une étagère, comme s'il assurait seul la transition du recto au verso.

Les teintes foncées dominant au recto de la jaquette, les pâles domineront à son verso. Le dessin général de la maquette pourrait être ainsi décrit: de vastes panneaux géométriques allant, 
au recto, du noir vers le blanc et couvrant à peu près l'entier de la jaquette, sont fissurés, à peu près en leur milieu mais davantage vers la gauche, par un couloir menant vers une porte dont on n'aperçoit qu'une faible portion. En fait, les hauts panneaux sont comme autant de murs séparant les pièces d'un appartement - et peuvent bien constituer la maquette du décor décrit en didascalie au début de la pièce:

Un seul décor représentant la salle commune d'un modeste appartement. [...] Une entrée principale et deux portes: l'une donne accès à la chambre d'Eulalie, l'autre ouvre sur un couloir qui dessert le reste de l'appartement. L'action se passe en une seule nuit (p. 14).

II y a nettement quelque chose d'écrasant, de vertigineux, pour parler comme Hector, dans ces hauts et sombres panneaux; et le triangle clair du plancher menant vers le minuscule rectangle blanc de la porte indique bien qu'ici on doit se faufiler vers la sortie, que les grands départs ne vont pas de soi, que les murs, même nus, retiennent: la hauteur de la porte (hauteur qu'on suppose établie en fonction de la taille humaine), par rapport à celle des murs qui l'encadrent, est déjà une indication que les départs rêvés ne sont pas faciles, que les murs finissent par prendre, sur les habitants d'un lieu, un ascendant considérable. Le temps de la pièce luimême, «une seule nuit», doit en principe mener de la noirceur à la clarté - clarté du jour, ou clarté consécutive aux difficiles épreuves de la nuit? Reprenant la même maquette (ou à peu près), mais en négatif et en miroir, le verso souligne aussi comme l'inverse mais pas tout à fait - de ce que nous venons d'affirmer: la clarté de la sortie est aussi la noirceur de l'inconnu, les hauts murs sombres devenant le blanc clair du trop connu - ces deux éléments pouvant aussi retenir et rendre les grands départs difficiles. "LES GRANDS DÉPARTS" en capitales blanches du recto, capitales claires en harmonie avec la clarté de la sortie entrevue, peuvent donc si aisément se muer en leur envers noir et à l'envers, en accord avec la sortie du verso.

Quelles couleurs ont donc le jour et la nuit, la réalité et les rêves de départ, dans quel sens même et selon quelle coloration faut-il lire les mots?

Prolongement du recto, le premier rabat présente une photo de Languirand - comme l'épine porte l'inscription de son nom; prolongement du verso, le deuxième rabat donne les crédits: «les photos qui illustrent le texte sont de André Le Coz», «la jaquette a été conçue par Jean-Claude Rinfret, et réalisée par Jean $\mathrm{Pa}$ quette», le tout suivi ici du nom de l'éditeur qui, comme celui de 
l'auteur, est absent du verso. Les deux rabats, en quelque sorte, célèbrent les créateurs: de la pièce et de son édition, l'auteur et ses metteurs en... livre. Et si le premier rabat est sur fond noir, le second est sur fond blanc: là aussi, symétrie et renversement. Géométrie, répétition, inversion, écho.

Or c'est précisément sur la parole d'un créateur, d'un romancier que s'ouvre la pièce en son incipit (résumé dans la première épigraphe): Hector, «au milieu de la pièce» (p. 15) en «désordre» (p. 14), dénudée, autant dire dans le chaos, se livre à «des essais de voix». II répète son nom que les murs lisses lui renvoient, en «écho». Il dira plus tard qu'il est "son plus fidèle auditeur» et sa femme lui demandera s'il cessera bientôt "de faire le perroquet». La voix qui s'essaie, la parole qui répète et se répète: ne pourraiton pas dire que le mot écho peut se lire dans le nom Hector? Roman (ou mythe) des origines, l'écho ce fut d'abord la nymphe Echo condamnée par Héra à ne plus se servir de la parole que pour répéter ce qui lui était dit: «Tu auras toujours le dernier mot, mais jamais plus tu ne parleras la première", avait proclamé l'épouse de Zeus. Dur châtiment pour l'amoureuse de Narcisse; sa vie durant, elle restera attachée à ce dernier, se consumant d'amour pour lui, devenant voix et voix seulement. Echo et Narcisse, Echo soeur de Narcisse selon Pausanias: écouter sa voix, regarder sa propre image. Ecouter sa propre voix redire son propre nom, c'est l'équivalent, sur le plan sonore, de la contemplation visuelle de soimême ${ }^{13}$. Et si le nom Hector, d'origine grecque, signifie «qui tient bon", le nom commun hector, en anglais, signifie fanfaron, matamore; et le verbe, dans la même langue, faire le fendant, tourmenter par les mots!

Ma foi, vous dites-vous, tout Hector est bien là: grand maître de la parole, fanfaron et fendant, il tourmente mots et phrases, cherchant à les lire et les dire en tous sens, l'endroit comme l'envers (puisque, grands départs ou pas, "nous sommes tous actuellement quelque part dans le monde», p. 67), cherchant leurs colorations, les embrouillant et les éclairant tout à la fois. II tourmente par les mots et par les phrases, poussant les êtres dans leurs derniers retranchements, forçant les aveux, obligeant à faire le départ entre le rêve et la réalité. En ce sens, Hector est bien celui "qui tient bon»: point d'ancrage de la pièce comme de la famille, il esquive et provoque, s'impose despotiquement/insolitement, oblige à la réflexion et au regard sans complaisance sur soi-même.

Maître du verbe auprès des siens, Hector ne réussit pourtant pas à maîtriser le verbe, son métier: romancier aux héros paralyti- 
ques, il recommence sans cesse des romans avortés qui semblent d'éternels échos d'eux-mêmes et de leur paralysie. Ce créateur de paralytiques - dans sa famille: son beau-père, comme dans ses oeuvres: ses héros - est un créateur paralysé: qui ne sait mener à terme l'acte d'écrire. Il y a chez Hector comme un écho d'Hamlet: la réflexion, dirait ce dernier, fait de lui un lâche, paralyse l'action. En ce sens, la fin des Grands départs peut se lire comme une revanche de la créature sur son créateur: le Grand-Père, paralytique depuis une querelle avec Hector, sera le seul à mener à terme un roman de ce dernier, à s'échapper des murs, à partir. Et le familial d'Hector, ainsi, investit sa création romanesque et lui montre la voie. Ou peut-être s'agit-il de la revanche du créateur sur sa créature: il est possible, signifie Languirand à Hector, de mener une oeuvre à terme grâce à un paralytique. Recto, verso, écho.

Hector-Écho et ses essais de voix ou de romans; HectorNarcisse et ses multiples reflets de lui-même dans l'appartement nu - car les siens, Albert y compris, sont bien à son image, à l'image de son verbe romancier: de même que les grands départs rêvés ne mènent toujours qu'aux petits retours hurniliés, cette parole semble celle de l'éternel retour du même, de dix ans en dix ans (p. 22 et 28), dans ses multiples variantes blanches et noires. En quoi la jaquette de couverture constitue bien une lecture attentive de l'oeuvre, à la fois dans ses mots, sa structure et ses significations.

La jaquette, le texte. Mais le paratexte renvoie aussi à d'autres éléments des parages, de “l'entourage" selon Genette ${ }^{14}$, immédiats du texte. Ainsi, à la page «Du même auteur» des Grands départs, il faudrait corriger les dates accolées au Roi ivre et au Hamlet d'après Kyd: ces deux pièces sont de 1956, non de 1957. La pièce est dédiée «à Louis-Georges Carrier, son ami, J.L. » (p. 7); la page précédente présentait justement, sous le nom de Jacques Languirand écrit en petites capitales, une brève biographie de J.L. commençant ainsi: «Animateur d'émissions de radio et de télévision. Et auteur dramatique», et rappelant ensuite les succès des Insolites et du Roi ivre - de toute évidence, cette note joue sur la notoriété médiatique de Languirand et ses succès scéniques tout récents. La page suivante ( $p .8$ ), qui donne les crédits de la création télévisée de l'oeuvre, se termine, forcément, sur «réalisation: Louis-Georges Carrier». Suivent, en hors-texte, quatre photos extraites de la réalisation télévisée - leur choix et leur ordonnance, que nous nous contenterons ici de décrire, pour- 
raient aussi constituer, à eux seuls, une lecture de l'oeuvre. La première montre Hector serrant sa fille dans ses bras; la seconde, un moment de l'affrontement Hector-Albert; la troisième, le départ d'Eulalie entourée de Hector et Margot; la quatrième, Hector et Margot qui, assis en face l'un de l'autre, sont aussi assis l'un à côté de l'autre tout en regardant, angoissés et dépités, dans des directions différentes.

Vient ensuite, en caractères italiques, un «Avant-propos» éditorial d'une page (p. 9), signé «Le Cercle du Livre de France». Cet avant-propos fait état de la réception critique de l'émission télévisée qui, dans une large mesure, explique/justifie la publication de l'oeuvre:

Lorsque les Grands Départs fut créé à la Télévision, le public manifesta avec une passion sans précédent dans les annales de la télévision canadienne, son approbation ou sa fureur. Des lettres, des coups de téléphone innombrables vinrent témoigner de toute une gamme de sentiments dont les extrêmes peuvent se résumer par «On n'a pas le droit de se moquer ainsi du public» et “Un chef-d'oeuvre enfin"s.

Louis-Georges Carrier dit plus loin ce qu'il pense de la pièce de Jacques Languirand. II précise qu'elle est pour lui avant tout une tragédie. Pour nous, c'est une comédie dont l'humour noir nous a séduit. Quant à l'auteur, il ne sait plus. (...).

Qui a raison? Les détracteurs des Grands Départs ou ses admirateurs? Ceux qui voient dans cette pièce une comédie [...], ou ceux pour qui elle est la tragédie [...]?

Le dernier mot reste toujours au public. C'est pourquoi nous publions les Grands Départs.

La publication rapide des Grands départs, donc, est largemnt liée à la controverse suscitée par la représentation télédiffusée de l'oeuvre; les pièces antérieures de Languirand, représentées à la scène, n'avaient sans doute pas eu, malgré leur succès, un aussi vaste auditoire. Et l'insolite d'un Hamlet d'après Kyd n'avait sans doute pas frappé les téléspectateurs. La controverse elle-même semble double: il y a les détracteurs et les admirateurs de la pièce d'une part, et chez ces derniers, d'autre part, les tenants de la tragédie et ceux de la comédie. Toujours: recto, verso, écho. La si rapide mais également si originale édition des Grands départs, en ces temps où les éditeurs québécois publiaient très parcimonieusement des pièces québécoises, a bien partie liée avec l'importance déjà grande de la Télévision (avec majuscule, comme dans l'avant-propos éditorial); elle s'appuie sur la controverse, bonne vendeuse, et lui fournit à la fois de quoi s'alimenter et de quoi rendre justice au texte; elle se présente également dans un décor visuel qui prolonge la représentation. 
Déjà annoncée dans l'avant-propos et lui faisant immédiatement suite, une brève analyse de l'oeuvre par son réalisateur, Louis-Georges Carrier. Analyse disposée sur deux colonnes en page 11, sur une portion de colonne (à peu près équivalente à celle qui, en page 6, présente l'auteur de la pièce) en page 10-comme si la mise en page de cette analyse avait été faite à partir de la fin: remplir la page 11 d'abord, puis reporter en page 10 cela qui reste. Le recto, quoi qu'on pense, peut parfois naître du verso.

Analyse brève, mais très riche et très articulée - le réalisateur est d'abord un liseur, un praticien du lisage, qui se double d'un traducteur: mettre en scène cela qui est en mot(s), re-présenter en images cela qui vous est présenté en mots. Quelques extraits de cette analyse, en commencant par son incipit:

Les Grands Départs est avant tout une tragédie qui se cache derrière le paravent de la comédie; une oeuvre en demi-teintes, recroquevillée sur elle-même comme une énigme [...].

Cette pièce fait le procès d'une société qui n'ose se regarder en face, qui joue à l'autruche dans les sables mouvants de l'inconscience. $(. .$.

Dans les Grands Départs, une terrible solitude referme les personnages sur eux-mêmes; [...] ils se regardent comme des choses transparentes [...].

[...]

Les Grands Départs pourrait se définir par la dualité du ridicule et du sublime qui se chevauchent tout au long de la pièce; de même que par un humour bien particulier dont le style de Languirand est toujours imprégné.

Ce style et cet humour, un ami très intime de Languirand et de Carrier, Hubert Aquin, à qui est dédié Les insolites, les avait déjà décrits dans le programme accompagnant les représentations de cette dernière (première?) pièce au Théâtre Anjou:

Un Languirand est un objet rare, aux contours fauves, de forme bizarre, dont les parois sont couvertes d'hiéroglyphes et qui tient, à la fois, de la boîte à surprise et de la pile électrique. [...].

On appelle aussi «languirand" un idiome qui s'apparente mystérieusement au moyen bengali et au turcoman. Racines grecques, grammaire siamoise, caractère plutôt chinois, tout, dans cette langue, semble concerté pour frapper violemment l'imagination.

Et qui dira, recto/verso, que l'on ne parle pas aussi de soi, parlant d'un autre? Narcisse, Hector le sait, n'est jamais bien loin. Écho non plus. Aquin, qui réalisera aussi avec Carrier plusieurs dramatiques télévisées; qui soumettra, à Carrier toujours, le projet d'un téléthéâtre ayant Hamlet pour sujet, projet qui finalement ne sera jamais réalisé ${ }^{15}$. Mais Hamlet se retrouvera dans Neige noire ${ }^{16}$, roman qui prend la forme d'un scénario - et dont l'un des person- 
nages principaux, Nicolas, est un créateur dont le narcissisme... et la quatrième de couverture de Neige noire présente en négatif une photo d'Aquin. Neige noire, blanc et noir, recto verso - mais sans doute sommes-nous là dans le para de la littérature, c'est-àdire dans son histoire ou ses origines; mais le para de la littérature ne pourrait-il pas déjà en être, comme le paratexte est bien du texte? Para-lisez.

1. Marthe Robert, Roman des origines et origines du roman (1972), coll. «Tel», no 13, Paris, Gallimard, 1976.

2. Jacques Languirand, Les grands départs, Montréal, le Cercle du Livre de France, 1958. Une deuxième édition des Grands départs sera publiée à Montréal en 1970 dans la collection "Lecture Québec" (dirigée par Serge Losique) aux Éditions du Renouveau pédagogique; présentée et annotée par l'auteur de cet article, cette édition contenait également une «mise en scène/ mise en place» de l'oeuvre par Languirand lui-même.

3. Jacques Languirand, Les insolites et Les violons de l'automne, Montréal, le Cercle du Livre de France, 1962, p. 8. Nous reviendrons, plus loin dans cet article, sur le fait que l'auteur du décor des Insolites a aussi conçu la jaquette des Grands départs; nous voulons signaler ici que la dessinatrice des costumes des Insolites, Yolande Delacroix-Pelletier, est aussi, depuis 1954, l'épouse de Languirand.

4. Ibid., p. 9.

5. On pourra lire à ce sujet notre article «Le Hamlet, adaptation d'après Thomas Kyd, de Jacques Languirand: "d'après Thomas Kyd", avez-vous dit?" (à paraître dans Voix \& images).

6. Jacques Languirand, Le dictionnaire insolite, Montréal, les Éditions du Jour, 1962.

7. Jacques Languirand, Le gibet, Montréal, le Cercle du Livre de France, 1960.

8. Gérard Genette, Palimpsestes. La Littérature au second degré, coll. "Poétique», Paris, Seuil, 1982 , p. 280 et ss.

9. La même remarque vaut aussi pour la première édition, cette même année 1958, d'une autre pièce importante de la dramaturgie québécoise, Un simple soldat de Marcel Dubé. Voir Marcel Dubé, Le temps des lilas et Un simple soldat, coll. «Théâtre canadien», tome I, Québec, Institut littéraire du Québec, 1958. Malgré l'ordonnance des titres sur l'épine de la jaquette, ordonnance qui correspond à l'ordre des pièces dans le volume, le recto de la jaquette affiche une photo de Gilles Pelletier dans le rôle du simple soldat, photo sur laquelle se superpose, en rouge, le titre Un simple soldat.

10. Gérard Genette, Seuils, coll. «Poétique», Paris, Seuil, 1987, p. 30.

11. Ibid.

12. Palimpsestes et Seuils de Genette contiennent, on le sait, de très nombreuses pages sur le rapport paratexte/métatexte.

13. Ces quelques lignes sur Narcisse et Écho sont tirées de notre article sur Les grands départs paru dans le Dictionnaire des oeuvres littéraires du Québec, tome III, Montréal, Fides, 1982 , p. 437.

14. Gérard Genette, Palimpsestes, p. 9.

15. Hubert Aquin, Point de fuite, Montréal, le Cercle du Livre de France, 1971, p. 141-142.

16. Hubert Aquin, Neige noire, Montréal, la Presse, 1974. 